\title{
DE MADRID AL CIELO: LA IMPORTANCIA DE ENFERMAR Y MORIR EN MADRID
}

RESUMEN. Madrid se ha convertido en una de las Comunidades Autónomas más saludables de España. Un factor fue la concentración de recursos que se produce, fundamentalmente, durante la década de los setenta. Pero el desarrollo del sistema sanitario durante esa década ha sido poco estudiado. El artículo analiza las relaciones entre el nivel de salud de la población de la CAM en esos años, la distribución de los recursos sanitarios y la disminución de las desigualdades sociosanitarias. Realiza un estudio comparativo entre la CAM y el resto de España, así como entre las ciudades de Madrid y Barcelona. Pone en duda las relaciones entre salud y recursos, así como entre el desarrollo económico y los progresos en salud pública. Al final se ofrecen diversas hipótesis que explican el buen nivel de salud de Madrid.

Se dice «De Madrid al cielo», con retintín, pero creyendo firmemente que en la capital de España se vive más y mejor ${ }^{1}$. Y es científicamente cierto. Quizá no sea ya debido a la mítica agua de Madrid, sino a la concentración de recursos, a la todavía abundante sangre nueva de inmigrantes que afluyeron a la Corte en los años sesenta, o al hecho de que casi nadie «se mata trabajando» (sic) en esa ciudad. En cualquier caso, vivir en Madrid - contra lo que algunos/as creen- protege de la enfermedad y ayuda a bien

${ }^{1}$ Este artículo no se hubiera escrito nunca si no hubiese sido por Carolina Mayeur. Muchísimas gracias, también, a la excelente ayuda técnica de Alicia Martino, Andrés Hevia y Rut V. Aguilera. Soy deudor de los consejos y críticas de Juan Salcedo, Josep A. Rodríguez, Mauro F. Guillén y Melissa G. Moyer. Gracias también al apoyo intelectual de Pedro Sabando. 
morir. Lejos ya, pues, de nosotros la idea de un Madrid culpable, azarzuelado y chulesco ${ }^{2}$, Madrid es un fenómeno sanitario, donde parece que da incluso gusto ponerse enfermo, y desde donde se va directamente al cielo (aunque el cielo últimamente parece ser que ya no se ve). Vale, pues, la pena que nos detengamos, aunque sea por unas hojas, a observar de cerca los procesos sociológicos, sanitarios y demográficos que durante las últimas décadas han convertido a Madrid en esa especie de paraíso terrenal. Una mirada microscópica nos demostrará que algunos problemas son mayores de lo que habíamos sospechado, y que como no se ponga remedio, pronto tendremos que escribir sobre el infierno de Madrid en el año 2000.

$\mathrm{Y}$ es que el crecimiento de la población en el caso de la Comunidad Autónoma de Madrid viene estrechamente aparejado al desarrollo económico. El período de máximo crecimiento coincide con la década del «milagro económico», 1960-1970:

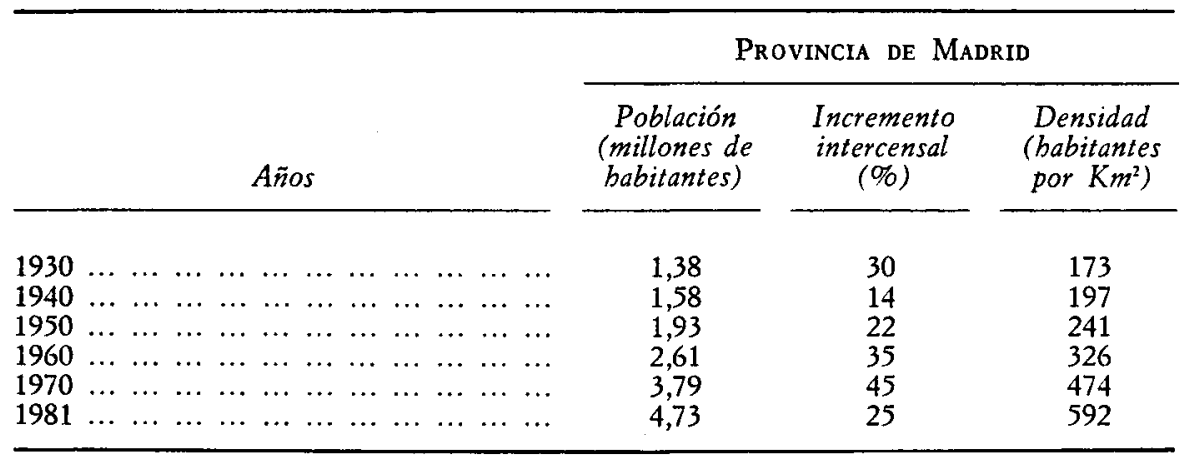

En los últimos años se produce - como causa de la crisis económica de finales de siglo - un estancamiento de la población, sobre todo de la capital. Según el Censo de 1981 (referido al día 1 de marzo), Madrid se convirtió por vez primera en la provincia más poblada de España, con una «población de hecho" de 4.726 .986 habitantes, o, lo que es lo mismo, el 12,5 por 100 de la población española total ( 37,7 millones). Es, además, una población con bastantes mujeres: 51,5 por 100 (frente al 50,9 por 100 del total nacional). Madrid-capital, la primera ciudad del país ( 1,8 veces más grande que Barcelona-capital), concentraba en 1981 aproximadamente las dos terceras partes de la población de toda la provincia (el 67,4 por 100 ), con un 52,5 por 100 de mujeres. En la década de los setenta (1970-1981) Madrid es la cuarta provincia que más aumenta de población, con un crecimiento intercensal del 25

${ }^{2}$ Claro que no me refiero aquí al estupendo libro de Juan Salcedo, Madrid culpable: Sobre el espacio y la población en las ciencias sociales (Madrid: Tecnos, 1977). 
por 100 , frente al 11 por 100 del total nacional. Sin embargo, la velocidad del crecimiento parece estar disminuyendo, pues entre 1975 y 1981 Madridprovincia creció sólo un 8,5 por 100 (el doble, de todas formas, del 4,6 por 100 de media nacional).

Madrid es una Comunidad Autónoma con un crecimiento vegetativo superior a la media nacional, con una tasa de natalidad relativamente alta, una de mortalidad más baja, y una de nupcialidad media. Según los datos para 1980, en Madrid nacieron 73.078 personas ( 200 al día) y fallecieron 28.974 ( 79 al día), lo que supone un saldo neto de 44.104 personas al año. Este saldo es el máximo provincial, con mucho, en España, sólo seguido de cerca por el saldo positivo de Barcelona, que es de 31.122 personas. A nivel de la población el saldo vegetativo de la provincia de Madrid es relativamente alto: el 0,9 por 100 (frente al 0,6 por 100 de Barcelona) y superior al 0,7 por 100 nacional. En Madrid sólo fallecen el 10,1 por 100 de los españoles/as que mueren (en 1980), y nacen el 12,9 por 100 de los españoles/as. Madrid-capital concentra casi todos los partos de la provincia (el 99 por 100), lo que facilita una buena planificación de los servicios públicos de asistencia al parto y maternidad, a la vez que permite reducir (o al menos controlar) la TMI. Las defunciones -lógicamente menos planificadas - no se concentran en la capital (hay unas pocas menos de las debidas según la población); y los matrimonios aún menos. En total, de los 73.078 nacimientos habidos en la provincia de Madrid, tan sólo 673 ocurrieron fuera de la capital $(0,9$ por 100). En lo que se refiere a partos, sólo Alcalá de Henares concentra un número no despreciable (estadísticamente), con 55 nacidos vivos en 1980. Los otros casos son menores incluso a 10 (Leganés, 4, Móstoles 3, Getafe 1). La provincia de Madrid (excluida la capital) se caracteriza por mantener un crecimiento vegetativo negativo, lo que supone una mortalidad dispersa y una natalidad muy concentrada.

Demográficamente, Madrid es una provincia muy similar a la media nacional, salvo que mantiene una tasa de mortalidad un poco más baja que la media nacional. De este hecho quizá se explique lo «de Madrid al cielo». Si la comparamos con Barcelona, la provincia catalana presenta una nupcialidad y natalidad bastante más bajas:

A ก̃ $O 1980$

Tasas por

\begin{tabular}{|c|c|c|c|c|c|}
\hline \multirow[b]{2}{*}{1.000 babitantes } & \multirow[b]{2}{*}{ España } & & \multirow[b]{2}{*}{ Madrid } & \multirow[b]{2}{*}{ Barcelona } \\
\hline & & Madrid & Barcelona & & \\
\hline $\begin{array}{llll}\text { Natalidad . .. } & \ldots & \ldots \\
\text { Mortalidad } & \ldots & \ldots & \ldots \\
\text { Nupcialidad } & \ldots & \ldots & \ldots\end{array}$ & $\begin{array}{r}15,1 \\
7,7 \\
5,7\end{array}$ & $\begin{array}{r}15,2 \\
6,0 \\
5,5\end{array}$ & $\begin{array}{r}12,8 \\
6,3 \\
4,2\end{array}$ & $\begin{array}{r}22,2 \\
7,3 \\
6,4\end{array}$ & $\begin{array}{r}20,5 \\
9,7 \\
4,1\end{array}$ \\
\hline
\end{tabular}


Todas las tasas son superiores si se refieren a la capital, conservando Madrid una ventaja clara en natalidad y nupcialidad, y una tasa de mortalidad más baja. Incluso a nivel de las 50 capitales de provincia, la tasa de mortalidad ( 9,0 por 1.000 habitantes) es superior a la de Madrid-capital (7,3).

La población madrileña se enfrenta con una serie de hechos al parecer irreversibles: familia nuclear, concentración de la población, desertización del interior, urbanización, secularización, liberación de la mujer, y revolución sexual. La nueva política sanitaria tiene que asumir esos procesos, reduciendo los aspectos negativos e interferencias que pudiesen haber. Los dos problemas que se observan cortan a la población por sus extremos: un paro creciente entre los jóvenes (mayor aún entre las mujeres) y un envejecimiento progresivo de la población. Este segundo proceso puede, sin embargo, disminuir algo en intensidad entre 1985 y el año 2000, debido a la estructura por edades de la población actual ${ }^{3}$.

La Comunidad Autónoma de Madrid no presenta una morbilidad hospitalaria demasiado alta. En total se hospitalizan cada año aproximadamente al 7,4 por 100 de los habitantes, frente al 8,6 por 100 del total nacional. Los tres sistemas o aparatos que -aparte de partos- generan más hospitalizaciones (y que son el digestivo, respiratorio, y cardiovascular o circulatorio) presentan en Madrid-provincia unas tasas sensiblemente inferiores:

\begin{tabular}{|c|c|c|c|}
\hline \multirow{3}{*}{$\begin{array}{l}\text { Hospitalizaciones debidas } \\
\text { al sistema o aparato }\end{array}$} & \multicolumn{3}{|c|}{$\begin{array}{l}\text { PACIENTES HOSPITALIZADOS EN } 1978 \\
\text { (por } 100.000 \text { habitantes) }\end{array}$} \\
\hline & \multirow{2}{*}{$\begin{array}{l}\text { Total de } \\
\text { España }\end{array}$} & \multicolumn{2}{|c|}{ Provincia de } \\
\hline & & Madrid & Barcelona \\
\hline $\begin{array}{llllllllll}\text { Digestivo } & \ldots & \ldots & \ldots & \ldots & \ldots & \ldots & \ldots & \ldots & \ldots\end{array}$ & 905 & 791 & 1.095 \\
\hline $\begin{array}{lllllllll}\text { Respiratorio } & \ldots & \ldots & \ldots & \ldots & \ldots & \ldots & \ldots & \ldots\end{array}$ & 640 & 500 & 1.014 \\
\hline $\begin{array}{llllllllll}\text { Cardiovascular } & \ldots & \ldots & \ldots & \ldots & \ldots & \ldots & \ldots & \ldots\end{array}$ & 498 & 427 & 612 \\
\hline
\end{tabular}

Además, si se compara Madrid con Barcelona se observa que las diferencias son más grandes todavía. Esto no significa necesariamente ni mejor ni peor situación sanitaria. Las provincias que en España hospitalizan a más del 10 por 100 de la población no son necesariamente las que tienen un nivel de salud más alto: Alava, Baleares, Burgos, La Coruña, La Rioja, Navarra, Sevilla, Zaragoza (aun cuando todas —a excepción de Sevilla- están en el norte del país).

En la tabla 1 se puede observar la distribución de enfermos en los hospitales (además de camas y estancias) según los tipos de asistencia. Cirugía,

${ }^{3}$ Para un análisis global del sistema sanitario español estoy tentado de recomendar el librito: Jesús M. DE Miguel, Estructura del sector sanitario (Madrid: Tecnos, 1983), 159 pp. En las pp. 149-155 hay una bibliografía básica seleccionada y anotada. 


\section{TABLA 1}

Enfermos/as atendidos en los bospitales en 1977

\begin{tabular}{|c|c|c|c|c|c|c|c|c|c|c|c|c|}
\hline \multirow[b]{3}{*}{ Tipos de asistencia } & \multicolumn{4}{|c|}{ Camas de hospitales } & \multicolumn{4}{|c|}{ ENFERMOS/AS INGRESADOS } & \multicolumn{4}{|c|}{ EstaNCIAS } \\
\hline & \multicolumn{2}{|c|}{ España } & \multicolumn{2}{|c|}{$\begin{array}{l}\text { Provincia } \\
\text { de Madrid }\end{array}$} & \multicolumn{2}{|c|}{ España } & \multicolumn{2}{|c|}{$\begin{array}{l}\text { Provincia } \\
\text { de Madrid }\end{array}$} & \multicolumn{2}{|l|}{ España } & \multicolumn{2}{|c|}{$\begin{array}{l}\text { Provincia } \\
\text { de Madrid }\end{array}$} \\
\hline & $N$ & $\%$ & $N$ & $\%$ & $N$ & $\%$ & $N$ & $\%$ & $N$ & $\%$ & $N$ & $\%$ \\
\hline 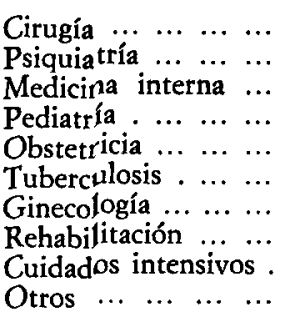 & $\begin{array}{r}57.245 \\
46.010 \\
37.521 \\
15.145 \\
14.781 \\
8.006 \\
6.156 \\
4.081 \\
3.878 \\
7.374\end{array}$ & $\begin{array}{r}28,6 \\
23,0 \\
18,7 \\
7,6 \\
7,4 \\
4,0 \\
3,1 \\
2,0 \\
1,9 \\
3,7\end{array}$ & $\begin{array}{l}8.368 \\
5.092 \\
6.040 \\
2.180 \\
1.601 \\
1.918 \\
868 \\
666 \\
718 \\
845\end{array}$ & $\begin{array}{r}29,6 \\
18,0 \\
21,3 \\
7,7 \\
5,6 \\
6,8 \\
3,1 \\
2,4 \\
2,5 \\
3,0\end{array}$ & $\begin{array}{r}1.260 .508 \\
76.005 \\
595.352 \\
304.112 \\
695.226 \\
17.408 \\
163.823 \\
18.365 \\
45.171 \\
65.579\end{array}$ & $\begin{array}{r}38,9 \\
2,3 \\
18,4 \\
9,4 \\
21,4 \\
0,5 \\
5,0 \\
0,6 \\
1,4 \\
2,0\end{array}$ & $\begin{array}{r}148.799 \\
13.188 \\
64.612 \\
39.961 \\
96.116 \\
3.291 \\
30.459 \\
4.614 \\
6.888 \\
9.011\end{array}$ & $\begin{array}{r}35,7 \\
3,2 \\
15,5 \\
9,6 \\
23,0 \\
0,8 \\
7,3 \\
1,1 \\
1,6 \\
2,2\end{array}$ & $\begin{array}{r}14.461 .156 \\
14.332 .445 \\
9.647 .528 \\
2.859 .862 \\
2.975 .712 \\
1.797 .385 \\
1.223 .336 \\
950.128 \\
340.141 \\
1.704 .317\end{array}$ & $\begin{array}{r}28,8 \\
28,5 \\
19,2 \\
5,7 \\
5,9 \\
3,6 \\
2,4 \\
1,9 \\
0,7 \\
3,4\end{array}$ & $\begin{array}{r}2.222 .783 \\
1.662 .934 \\
1.524 .332 \\
471.995 \\
383.948 \\
410.473 \\
223.433 \\
158.880 \\
55.479 \\
168.906\end{array}$ & $\begin{array}{r}30,5 \\
22,8 \\
20,9 \\
6,5 \\
5,3 \\
5,6 \\
3,1 \\
2,2 \\
0,8 \\
2,3\end{array}$ \\
\hline $\operatorname{ToTAL} \ldots \ldots \ldots$ & 200.197 & 100 & 28.296 & 100 & 3.241 .549 & 100 & 416.939 & 100 & 50.292 .010 & 100 & 7.283 .163 & 100 \\
\hline
\end{tabular}

FuENTE: INE, Anuario Estadístico de España 1981 (Madrid: INE, 1982), pp. 649-652. 
medicina interna y partos superan juntos el 79 por 100 de los entermos ingresados en hospitales en España, pero sólo el 55 por 100 de las camas, dado que son enfermos de estancias no muy largas. Psiquiatría, por ejemplo, que ocupa casi la cuarta parte de las camas (el 23 por 100), no ingresa más que el 2,3 por 100 de los pacientes. La provincia de Madrid mantiene una estructura de morbilidad hospitalaria similar a la nacional, sin notables diferencias, salvo ingresar más pacientes psiquiátricos y ginecológicos, a pesar de no tener más camas de esos tipos, sino incluso menos. Desde la perspectiva de las estancias - que son las que realmente sirven para planificar los recursos necesarios - la psiquiatría recupera su importancia (con el 29 por 100 de las estancias a nivel nacional y 23 por 100 de la provincia de Madrid), aunque se señala la mayor productividad del dispositivo hospitalario psiquiátrico.

En lo que se refiere a ingresos hospitalarios, más de la tercera parte de los ingresos de las mujeres se refieren al ciclo reproductor: parto (normal y complicado), complicaciones del embarazo y del puerperio, y «aborto». En el total nacional esos casos representan el 38 por 100 de las hospitdlizaciones femeninas, y en Madrid-provincia alcanzan el 42 por 100. En todas las otras causas de morbilidad (según la Encuesta de Morbilidad Hospitalaria de 1978) la mujer se hospitaliza menos que el varón, a excepción de unas pocas causas: tumores, glándulas endocrinas, y envenenamiento. En algunos casos la sobremorbilidad masculina es alta: 2,6 veces más varones por tuberculosis, 1,8 veces por alteraciones mentales, 2,0 veces por anomalías congénitas, 1,6 veces por fracturas, 2,3 por luxaciones, 2,4 por traumatismos internos, 3,2 por heridas, y 2,2 otros accidentes. En el caso de Madrid las diferencias son aún mayores en ciertos casos: 2,4 por anomalías congénitas, 2,8 por luxaciones, y 4,4 por heridas. Sin embargo, en el contexto global (y debido a la sobremorbilidad asociada a la reproducción) en España, en 1978, el 56 por 100 de las personas hospitalizadas son mujeres; y en Madrid-provincia se eleva al 59 por 100 .

Los procesos de fecundidad es la causa más normal de hospitalización en nuestro país, alcanzando al 2 por 100 de todos los habitantes, cada año. Si tenemos en cuenta sólo a las mujeres, supone el 3,7 por 100 de hospitalizaciones al año. Madrid-provincia mantiene una tasa alta, con una proporción relativamente alta de «abortos» (casi el doble de la de Barcelona-provincia). La tasa correspondiente a «abortos» incluye tanta variación que todo hace sospechar que incluye abortos voluntarios. Si esto es así es muy posible que entre los 223 «abortos» por 100.000 habitantes al año conviniese realizar un estudio en profundidad (lo que no sugerimos es que se reduzcan; incluso podría ser conveniente que aumentasen, si con ello mejora la salud de algunas mujeres). En cualquier caso hay que notar que el 78 por 100 de las hospitalizaciones por procesos de fecundidad no se refieren a ningún problema ni enfermedad, sino tan sólo a parto normal. Es por ello por lo que no se 
puede hablar de «enfermos/as», sino de «pacientes» o, mejor, de «población hospitalizada». La «morbilidad» del ciclo reproductor -típicamente femenina - incluye un porcentaje estimable de hospitalizaciones femeninas debidas meramente al parto normal $(30$ por 100$)$, en 1978 :

\begin{tabular}{|c|c|c|c|c|c|}
\hline & \multicolumn{2}{|c|}{ EsPaÑA } & \multicolumn{3}{|c|}{ Provincia de Madrid } \\
\hline & $N$ & $\%$ & $N$ & $\%$ & $\begin{array}{c}\text { Estancias } \\
\text { medias }\end{array}$ \\
\hline 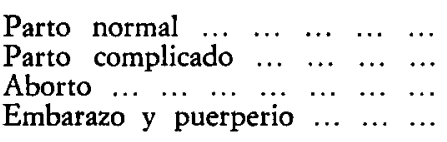 & $\begin{array}{r}531.326 \\
50.595 \\
76.112 \\
28.799\end{array}$ & $\begin{array}{r}77,4 \\
7,4 \\
11,1 \\
4,2\end{array}$ & $\begin{array}{r}74.035 \\
5.816 \\
10.268 \\
2.110\end{array}$ & $\begin{array}{r}80,3 \\
6,3 \\
11,1 \\
2,3\end{array}$ & $\begin{array}{l}4,0 \\
7,8 \\
2,8 \\
3,4\end{array}$ \\
\hline $\begin{array}{lllllll}\text { TOTAL } & \ldots & \ldots & \ldots & \ldots & \ldots & \ldots\end{array}$ & 686.832 & 100 & 92.229 & 100 & 4,1 \\
\hline Hospitalizaciones de mujeres $\ldots$ & 1.782 .417 & $(38,5)$ & 221.117 & $(41,7)$ & 12,6 \\
\hline
\end{tabular}

En Madrid la patología es menor, y la mayoría son partos normales. Por otro lado, las mujeres se hospitalizan menos por otras causas. Las hospitalizaciones por reproducción son muy cortas (cuatro días de media), frente a los trece días de media para el total de hospitalizaciones.

Los casos de enfermedades infecciosas y parasitarias (descontados los casos perinatales) representan más hospitalizaciones que la morbilidad por cáncer, con más de 23 casos por 10.000 habitantes en 1978. Madrid-provincia mantiene una tasa bastante inferior a la media (menos de 20 casos por cada 10.000 habitantes), y mucho menor que Barcelona (con 24 casos). Sin embargo, tres provincias de la dasicora (Segovia, Guadalajara y Avila) mantienen tasas altas. Los casos víricos son muy comunes en Madrid, así como los de hidatidosis en toda la dasicora madrileña. Las estadísticas señalan en general la necesidad de programas especiales en enfermedades víricas y en infecciosas en general.

En algunos aspectos importantes la morbilidad de la CAM parece estar bien atendida. Un caso claro es del cáncer. En el contexto del total de España se hospitalizan aproximadamente cada año el mismo número de personas por cáncer que personas mueren de cáncer en el país (aun cuando de esos hospitalizados sólo mueran el 10 por 100 dentro del hospital). En Madrid-provincia, sin embargo, se hospitalizan muchas más personas de las que mueren: 
PACIENTES CON CÁNCER EN 1978

\begin{tabular}{|c|c|c|c|c|}
\hline & \multirow{2}{*}{\multicolumn{2}{|c|}{ Varones }} & & \\
\hline & & & \multicolumn{2}{|c|}{ Mujeres } \\
\hline & Mueren & $\begin{array}{c}\text { Se bospi- } \\
\text { talizan }\end{array}$ & Mueren & $\begin{array}{c}\text { Se bospi- } \\
\text { talizan }\end{array}$ \\
\hline $\begin{array}{lllllllll}\text { España } & \ldots & \ldots & \ldots & \ldots & \ldots & \ldots & \ldots & \ldots \\
\text { Madrid-provincia } & \ldots & \ldots & \ldots & \ldots & \ldots \\
\text { Barcelona-provincia } & \ldots & \ldots & \ldots & \ldots \\
\end{array}$ & $\begin{array}{r}32.293 \\
2.886 \\
3.950\end{array}$ & $\begin{array}{r}29.410 \\
5.575 \\
3.985\end{array}$ & $\begin{array}{r}23.751 \\
2.211 \\
3.023\end{array}$ & $\begin{array}{r}29.205 \\
6.039 \\
3.445\end{array}$ \\
\hline
\end{tabular}

Así, la CAM concentra aproximadamente el 20 por 100 de las hospitalizaciones de cáncer de todo el país; más aún en el caso de las mujeres:

\begin{tabular}{|c|c|c|c|}
\hline \multirow[b]{2}{*}{ Tumores malignos } & \multicolumn{2}{|c|}{$\begin{array}{c}\text { CASOS hospiTALIZADOS } \\
\text { EN } 1978\end{array}$} & \multirow[b]{2}{*}{$\%$} \\
\hline & España & $\begin{array}{l}\text { Madrid. } \\
\text { provincia }\end{array}$ & \\
\hline 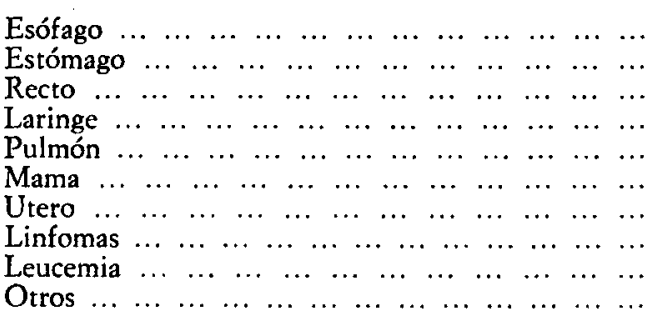 & $\begin{array}{r}687 \\
2.295 \\
1.083 \\
2.197 \\
4.070 \\
8.036 \\
1.390 \\
5.793 \\
4.526 \\
28.536\end{array}$ & $\begin{array}{r}157 \\
342 \\
279 \\
629 \\
898 \\
2.210 \\
437 \\
825 \\
548 \\
5.289\end{array}$ & $\begin{array}{l}23 \\
15 \\
26 \\
29 \\
22 \\
28 \\
31 \\
14 \\
12 \\
18\end{array}$ \\
\hline
\end{tabular}

Los cánceres más tradicionales (como el de estómago) sólo representan el 15 por 100 del total de casos en España, superior a la media; pero el de útero (en su mayoría cuello uterino) son casi la tercera parte. En resumen, todos los datos indican que Madrid está haciendo un esfuerzo considerable por tratar las morbilidades crónicas más complicadas.

La Comunidad Autónoma de Madrid concentra también muchos casos de morbilidad mental; pues con el 12,5 por 100 de población española hospitaliza al 16 por 100 de los pacientes con supuestas «alteraciones mentales». Parte de ese exceso se debe a la abundancia de drogadicciones, incluyendó alcoholismo: 


\begin{tabular}{|c|c|c|c|c|c|}
\hline \multirow[b]{2}{*}{ Alteraciones mentales } & \multicolumn{3}{|c|}{$\begin{array}{l}\text { NÚMERO DE CASOS } \\
\text { HOSPITALTZADOS } \\
\text { EN } 1978\end{array}$} & \multicolumn{2}{|c|}{$\begin{array}{c}\text { PROPORCIÓN } \\
\text { DE VARONES (\%) }\end{array}$} \\
\hline & España & $\begin{array}{c}\text { Madrid- } \\
\text { provincia }\end{array}$ & $\%$ & España & $\begin{array}{l}\text { Madrid- } \\
\text { provincia }\end{array}$ \\
\hline 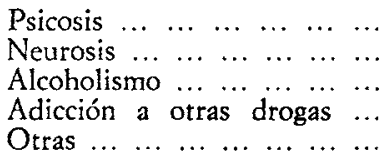 & $\begin{array}{r}30.158 \\
10.979 \\
15.990 \\
1.320 \\
8.865\end{array}$ & $\begin{array}{r}4.174 \\
1.912 \\
2.853 \\
341 \\
1.295\end{array}$ & $\begin{array}{l}14 \\
17 \\
18 \\
26 \\
15\end{array}$ & $\begin{array}{l}54 \\
56 \\
86 \\
79 \\
71\end{array}$ & $\begin{array}{l}52 \\
53 \\
86 \\
80 \\
58\end{array}$ \\
\hline $\begin{array}{llllll}\text { TotaL } & \ldots & \ldots & \ldots & \ldots & \ldots\end{array}$ & 67.312 & 10.575 & 16 & 65 & 63 \\
\hline
\end{tabular}

Los varones suponen generalmente casi las dos terceras partes de los pacientes mentales (un porcentaje que es bastante mayor en drogadicciones). Madrid-provincia mantiene una proporción de varones algo inferior a la media (63 por 100), pero todavía superior a Barcelona-provincia (61 por 100). Hay que tener en cuenta (no hay datos para Madrid, pero no serían muy diferentes) que de las personas hospitalizadas por alteraciones mentales tan sólo el 4,4 por 100 ingresan por petición propia. El 65,5 por 100 son ingresados por orden del médico, el 19,4 por 100 por petición familiar, y el 4,9 por 100 por orden gubernativa o judicial.

La mortalidad por causa externa de lesión (es decir, accidentes, suicidio, y homicidio fundamentalmente) supone entre el 5 y el 6 por 100 de todos los fallecidos cada año. Se empieza, pues, a considerar como la tercera causa general de muerte. Contrario a lo que se podía suponer de entrada, la mortalidad por causa externa es menor en Madrid ( obre todo en la capital) que en el resto de España. En parte se explica porque la mortalidad causada por vehículos de motor (a pesar de la densidad circulatoria) tiene la mitad de importancia que en el resto de España; lo mismo con el suicidio. Sin embargo, los demás accidentes (envenenamiento, caídas, fuegos, sumersión, armas, industriales) son bastante más numerosos. En Madrid también la proporción de varones es un poco menor, aunque todavía representa los dos tercios. La sobremortalidad masculina por causa externa es especialmente alta en homicidios (siempre por encima del 80 por 100 de varones) y en suicidios (alrededor del 70 por 100 de varones). Una campaña de prevención debería, pues, tener en cuenta esas diferencias. Las fracturas de todo tipo suponen aproximadamente un 4 por 100 de todas las hospitalizaciones en España. Contrario a lo que se puede suponer a primera vista, ese porcentaje es menor en las grandes capitales: 3,5 por 100 en Barcelona-provincia, y 2,8 por 100 en Madrid-provincia. Es una morbilidad típicamente masculina (superior al 60 por 100 de varones). En total, los accidentes, envenenamientos y violencias suponen una de cada diez hospitalizaciones en España en la 
TABLA 2

Pacientes atendidos en bospitales a través del INSALUD, en Madrid-provincia, en 1980, para intervenciones quirúrgicas

\begin{tabular}{|c|c|c|c|c|c|c|c|}
\hline \multirow[b]{2}{*}{ Tipos de asistencia } & \multicolumn{2}{|c|}{$\begin{array}{l}\text { INTERVENCIONES EN } \\
\text { CONSULTAS EXTERNAS }\end{array}$} & \multicolumn{2}{|c|}{$\begin{array}{l}\text { ENFERMOS/AS } \\
\text { INGRESADOS }\end{array}$} & \multicolumn{2}{|c|}{ ESTANCIAS } & \multirow{2}{*}{$\begin{array}{l}\text { Dias de } \\
\text { estancia }\end{array}$} \\
\hline & $N$ & $\%$ & $N$ & $\%$ & $N$ & $\%$ & \\
\hline 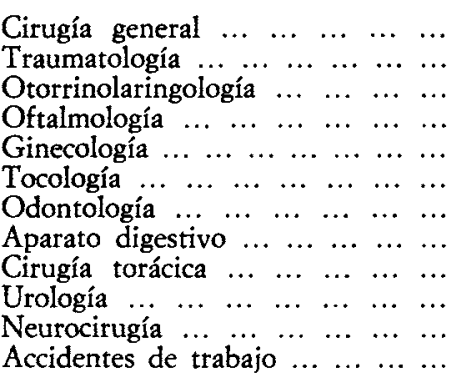 & $\begin{array}{l}14.210 \\
31.214 \\
2.297 \\
9,800 \\
2.140 \\
- \\
188 \\
347 \\
8.685 \\
4.505 \\
2.777 \\
-\end{array}$ & $\begin{array}{r}18,6 \\
41,0 \\
3,0 \\
12,9 \\
2,8 \\
- \\
0,2 \\
0,4 \\
11,4 \\
5,9 \\
3,6 \\
\end{array}$ & $\begin{array}{r}26.680 \\
15.261 \\
4.709 \\
5.556 \\
8.513 \\
49.907 \\
2.035 \\
4.572 \\
6.898 \\
5.218 \\
2.905 \\
875\end{array}$ & $\begin{array}{r}20,0 \\
11,5 \\
3,5 \\
4,2 \\
6,4 \\
37,5 \\
1,5 \\
3,4 \\
5,2 \\
3,9 \\
2,2 \\
0,6\end{array}$ & $\begin{array}{r}298.950 \\
257.721 \\
37.688 \\
57.265 \\
92.262 \\
245.073 \\
31.423 \\
112.805 \\
104.731 \\
72.062 \\
56.166 \\
15.105\end{array}$ & $\begin{array}{r}21,6 \\
18,6 \\
2,7 \\
4,1 \\
6,6 \\
17,7 \\
2,3 \\
8,2 \\
7,6 \\
5,2 \\
4,1 \\
1,1\end{array}$ & $\begin{array}{r}11,2 \\
16,9 \\
8,0 \\
10,3 \\
10,8 \\
4,9 \\
15,4 \\
24,7 \\
15,2 \\
13,8 \\
19,3 \\
17,3\end{array}$ \\
\hline Total $\ldots \ldots \ldots \ldots$ & 76.163 & 100 & 133.129 & 100 & 1.381 .251 & 100 & 10,4 \\
\hline
\end{tabular}

Nota: Incluye todas las llamadas residencias sanitarias, y clínicas quirúrgicas concertadas. Fuente: INE, Anuario Estadístico de España 1981 (Madrid: INE, 1982), pp. 657-663. 
actualidad. Una causa de morbilidad accidental, sospechosa de contener algunos suicidios encubiertos, es la de «efectos adversos de sustancias de acción medicinal». Una parte de los casos se concentran en el grupo de edad de uno a cuatro años y suponemos que son realmente accidentes. Otra gran cantidad de casos (la más numerosa) se concentra entre los quince y los veinticuatro años, y particularmente en mujeres:

\begin{tabular}{|c|c|c|}
\hline & \multicolumn{2}{|c|}{$\begin{array}{l}\text { ENVENENAMIENTO CON } \\
\text { MEDICINAS EN } 1978\end{array}$} \\
\hline & $N .^{\circ}$ de casos & $\%$ mujeres \\
\hline 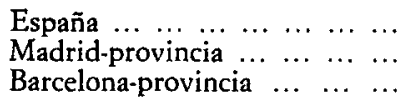 & $\begin{array}{l}9.704 \\
1.093 \\
1.273\end{array}$ & $\begin{array}{l}67 \\
72 \\
72\end{array}$ \\
\hline
\end{tabular}

Una alta proporción de la atención sanitaria realizada por los hospitales de la Seguridad Social (o concertados) son intervenciones quirúrgicas, con una estancia media de diez días, aproximadamente la mitad del total de estancias hospitalarias. En la tabla 2 se puede ver las operaciones realizadas en Madrid, en 1980, por especialidades. Más de la tercera parte (el 38 por 100) son partos, aunque sólo ocupan el 5 por 100 de las camas totales. Su incidencia es importante, pues se atienden aproximadamente a las dos terceras partes de los partos a través del INSALUD, concentrando casi la quinta parte de los recursos hospitalarios. Una quinta parte adicional es cirugía general. Indirectamente se pueden así evaluar las pautas de morbilidad de la población, y sus necesidades. En total suponen 29 días de estancia al año en hospitales (a través del INSALUD) por cada 100 madrileños.

La mortalidad de la Comunidad Autónoma de Madrid es muy baja (apenas seis muertos por cada 1.000 habitantes en 1978). Esta mortalidad es aún más baja, casi la mitad, en los municipios industriales en torno de la capital, debido a una estructura de edades muy joven. La mortalidad general ha descendido considerablemente en Madrid-capital, desde casi el 3 por 100 a principios de siglo:

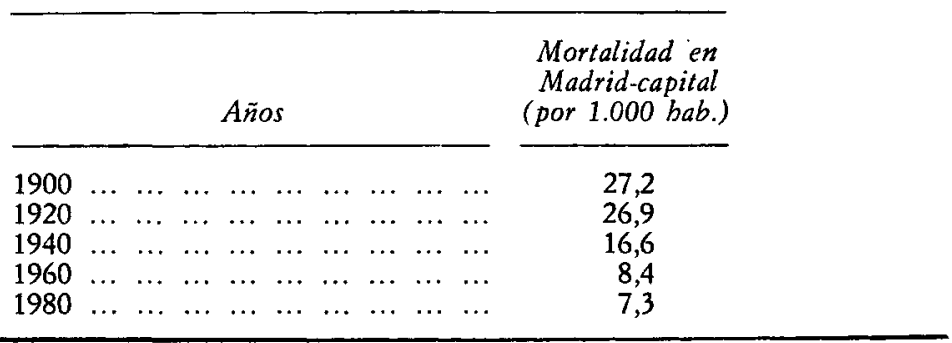


No parece que vaya a descender mucho más, pues es ya baja para una gran ciudad como Madrid.

En la CAM mueren aproximadamente 28.235 personas (es decir, unas 77 personas al día). La mortalidad infantil supone el 3,4 por 100 del total, siendo más común entre los varones (3,8 por 100) que entre las mujeres $(3,0$ por 100$)$. En general, mueren - lo mismo que nacen- 109 varones por cada 100 mujeres. Las dos terceras partes de las defunciones son personas de sesenta y cinco y más años:

\begin{tabular}{|c|c|c|c|}
\hline & \multicolumn{3}{|c|}{ \% FALLECIDOS EN 1978} \\
\hline & $\begin{array}{c}\text { Menores de } \\
1 \text { año }\end{array}$ & $\begin{array}{c}\text { De } 65 \text { años } \\
y \text { más }\end{array}$ & $\begin{array}{c}\text { De } 85 \text { años } \\
y \text { más }\end{array}$ \\
\hline 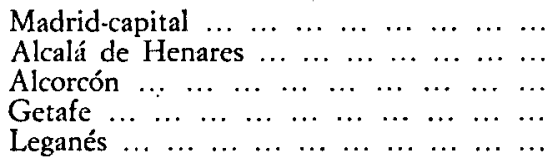 & $\begin{array}{l}3,4 \\
2,9 \\
5,0 \\
3,8 \\
6,2\end{array}$ & $\begin{array}{l}67,0 \\
65,5 \\
56,4 \\
56,3 \\
54,9\end{array}$ & $\begin{array}{r}14,5 \\
15,4 \\
9,3 \\
10,0 \\
8,8\end{array}$ \\
\hline TOTAL de la PROVINCIA $\ldots \ldots \ldots \ldots$ & 3,4 & 66,6 & 14,5 \\
\hline
\end{tabular}

Como se puede observar, la mortalidad se distribuye mejor en Alcalá de Henares (poca mortalidad infantil, y muchos que mueren a los ochenta y cinco años y más). El resto del cinturón industrial de Madrid-ciudad está peor incluso que la capital. Obviamente, estos datos dependen de la estructura de edades de la población; un dato que para 1978 es imposible de evaluar bien, dado que no se tenían los datos completos del Censo de 1981.

Las tres causas de muerte principales suponen al menos las dos terceras partes de los fallecidos: cardiovasculares (que se acerca a la mitad de los casos), cáncer (que es un caso de cada cinco), y accidentes (en torno al 5 por 100 de los casos). Las tres son causas difíciles de evitar en un mundo moderno:

\begin{tabular}{|c|c|c|c|}
\hline \multirow[b]{2}{*}{ Causas de fallecimiento } & \multicolumn{3}{|c|}{ MADRID EN $1978(\%)$} \\
\hline & Total & Capital & $\begin{array}{l}\text { Resto de la } \\
\text { provincia }\end{array}$ \\
\hline $\begin{array}{lcccccccccc}\text { Cardiovasculares } & \ldots & \ldots & \ldots & \ldots & \ldots & \ldots & \ldots \\
\text { Cáncer } & \ldots & \ldots & \ldots & \ldots & \ldots & \ldots & \ldots & \ldots & \ldots & \ldots \\
\text { Accidentes } & \ldots & \ldots & \ldots & \ldots & \ldots & \ldots & \ldots & \ldots & \ldots\end{array}$ & $\begin{array}{r}48,7 \\
19,0 \\
5,2\end{array}$ & $\begin{array}{r}43,2 \\
19,1 \\
4,6\end{array}$ & $\begin{array}{r}69,7 \\
18,9 \\
7,3\end{array}$ \\
\hline $\begin{array}{rllllllll}\operatorname{Total} & \ldots & \ldots & \ldots & \ldots & \ldots & \ldots & \ldots & \ldots \\
\text { (Causas clasificables) } & \ldots & \ldots & \ldots & \ldots & \ldots & \ldots\end{array}$ & $\begin{array}{c}72,9 \\
(26.746)\end{array}$ & $\begin{array}{c}66,9 \\
(21.220)\end{array}$ & $\begin{array}{c}95,9 \\
(5.526)\end{array}$ \\
\hline
\end{tabular}


Curiosamente, la incidencia de estas tres grandes causas es mayor en el resto de la CAM que en la capital. La causa es la importancia de las enfermedades cardiovasculares en la zona rural y semiurbana; así como una tasa elevada de accidentes.

Del embarazo, parto y puerperio murieron en Madrid-provincia cinco mujeres (cuatro de ellas en Madrid-capital). Esta cantidad es «pequeña», incluso si la comparamos con los 82 casos en toda España (suponen sólo el 6 por 100). Sin embargo, convendría tomar todo tipo de medidas para reducir aún más ese número. Una posibilidad sería obligar a realizar un informe especial sobre cada uno de esos casos, e investigar detenidamente las causas y responsabilidades. Otra medida preventiva es establecer una asistencia pública prenatal de calidad. Este es uno de los problemas básicos de la atención a la fecundidad en Madrid, ya que es un sector de mala calidad y privatizado.

Todavía las enfermedades infecciosas y parasitarias suponen en Madridprovincia el 2,2 por 100 de casos. En cualquier caso esa proporción es ligeramente superior a la media nacional, que es el 2,1 por 100 de los casos. Todo sugiere que estamos ante un proceso de incremento de las enfermedades infecciosas y parasitarias, lo que supone un retroceso considerable del nivel de salud de la población. Las enfermedades del sistema respiratorio son una causa importante de mortalidad, suponiendo el 8,2 por 100 de las muertes de la provincia de Madrid a finales de la década de los setenta. A pesar de la supuesta polución de la ciudad de Madrid, y de su contaminación, la mortalidad respiratoria no es más que el 4,8 por 100 del total. Sólo la cirrosis hepática supone 873 muertes (en 1978), o, lo que es lo mismo, el 3,3 por 100 del total de fallecidos (el 3,6 en Madrid-capital). Su relación con el consumo de alcohol sugiere la necesidad de crear un programa especial para intentar reducir esta causa de mortalidad, especialmente en la capital.

El cáncer es una causa creciente de muerte, que en la provincia (y la capital) de Madrid supone ya el 19 por 100 de los fallecimientos. Todavía la primera causa es cáncer del aparato gastrointestinal (1.290 casos en 1978), el 52 por 100 de los cuales se refieren al estómago. Algunos casos gastrointestinales se relacionan también con el tabaco. Sin embargo, la causa simple más importante es el cáncer de pulmón -estructuralmente asociado con el consumo de cigarrillos-, con 718 casos. El total del aparato respiratorio supone el 27 por 100 de los cánceres clasificables. El tercer cáncer en importancia es el de mama, con el 10 por 100 de los casos totales, y la cuarta parte de las mujeres que mueren de cáncer. Las diferencias por sexo son llamativas; la mujer muere relativamente más de cánceres gastrointestinales (la mujer es la que usualmente cocina) y los varones de cáncer del sistema respiratorio (los varones fuman tabaco mucho más que las mujeres, aun cuando las diferencias se van reduciendo). Lo que es impresionante es que todavía mueren al año 337 mujeres de cáncer de mama (el 25 por 100 de 
los casos de mujeres), y 139 de cáncer de útero (el 10 por 100 de esas mujeres), muchos de los cuales serían evitables con un programa de atención sanitaria adecuada. El cáncer es uno de los problemas sanitarios en los que una política de salud adecuada podría producir avances más espectaculares.

$\mathrm{Si}$ se analiza la proporción de fallecidos de cáncer por diversos tipos (los más prevenibles) y grupos de edad, se observa que esos datos son esenciales para planificar un programa adecuado de prevención de diversos tipos de cáncer en la Comunidad Autónoma de Madrid. Así, la mayoría de los cánceres empiezan a ser mortales a partir de los cuarenta años de edad, aunque se gestan a veces dos décadas antes (como el cáncer de pulmón, relacionado con el consumo de tabaco). El screening de cáncer de mama, según las estadísticas, debería de empezar a los veinticinco años o antes; y lo mismo el de útero y recto. Ello supone establecer nuevas conductas médicas, y de autoexploración de la población en algunos casos. En los casos de pulmón y estómago (desgraciadamente los más abundantes) no queda más remedio que reducir o eliminar las causas. La reducción drástica en el consumo de tabaco entre los/as jóvenes y el control riguroso de la industria alimenticia son dos objetivos precisos a implementar lo antes posible en la CAM.

España, como es sabido, mantiene una alta tasa de accidentes de trabajo en el contexto europeo. Afortunadamente, en la década que aquí analizamos (1971-1980), el número absoluto de accidentes de trabajo se han reducido aproximadamente a la mitad (al 52 por 100). Aunque Madrid mantiene una proporción relativamente baja ( 10,2 por 100 en 1980), es importante notar que ese porcentaje ha subido (9,7 por 100 en 1971), en vez de disminuir. En números absolutos supuso casi 60.000 accidentes de trabajo en 1980. En Barcelona el número es mucho más alto, alrededor de 106.000 (el 18 por 100 de todos los accidentes de trabajo en España), aunque están disminuyendo. En lo que se refiere al total de accidentes de trabajo, España ha reducido su número ( $\mathrm{y}$ tasa) a la mitad en la última década. En 1980 hubieron casi 600.000 accidentes de trabajo, lo que representa un 5 por 100 de la población activa; y un 10 por 100 se produjeron en Madrid-provincia. No sólo hay menos accidentes en Madrid-provincia, sino que son menos mortales o graves (el 1,8 por 100). Los accidentes de circulación en 1980 fueron casi 100.000 en toda España, y sólo 8,8 en Madrid-provincia. Por cada 100 accidentes, hubieron 5,3 personas muertas en España, y sólo 4,4 en Madrid-provincia:

\begin{tabular}{|c|c|c|c|}
\hline \multirow[b]{2}{*}{ Accidentes de circulación } & \multicolumn{3}{|c|}{$\begin{array}{c}\text { TASA DE ACCIDENTADOS EN } 1980 \\
\text { (por } 100 \text { habitantes) }\end{array}$} \\
\hline & España & $\begin{array}{l}\text { Madrid- } \\
\text { provincia }\end{array}$ & $\%$ \\
\hline 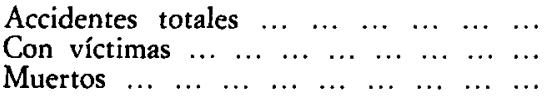 & $\begin{array}{c}100 \\
72 \\
5,3\end{array}$ & $\begin{array}{c}100 \\
77 \\
4,4\end{array}$ & $\begin{array}{l}8,8 \\
9,4 \\
7,2\end{array}$ \\
\hline
\end{tabular}


En resumen, en la CAM hay relativamente pocos accidentes de circulación, pero suelen haber más víctimas, aunque menos mortales. En cualquier caso, en 1980 nada menos que 361 personas murieron en Madridprovincia causados por accidentes de circulación; es decir, prácticamente una persona diaria (frente a 5.017, ó 14 personas diarias en toda España). Los accidentes de circulación son un coste considerable, aunque la proporción de víctimas en Madrid no es relativamente muy alto. La CAM concentra el 9,6 por 100 de los accidentados, y el 7,2 por 100 de los muertos en accidentes de circulación. Sin embargo, la proporción de vehículos matriculados en 1980 fue del 15,5 por 100, y la proporción de permisos de conducir expedidos, del 13,0 por 100 . En cualquier caso, las estadísticas muestran aproximadamente un muerto/a diario.

Si se mide la distribución por grupos de edades de la mortalidad debida a causa externa en la CAM, en 1978, se observa que la pauta general es una concentración de edades maduras, con pocos casos en los menores de cinco años y pocos también en los mayores de sesenta y cinco años. La única excepción a esta pauta es el suicidio, que, como es sabido, suele aumentar con la edad. La proporción de varones es alrededor de los dos tercios de los casos por lo menos, pero con diferencias:

\begin{tabular}{|c|c|}
\hline Tipos de accidentes & $\begin{array}{l}\text { \% varones } \\
\text { del total } \\
\text { de fallecidos }\end{array}$ \\
\hline 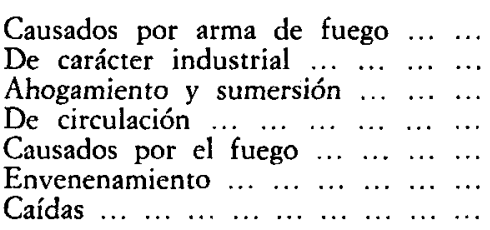 & $\begin{array}{l}87 \\
81 \\
74 \\
71 \\
65 \\
56 \\
52\end{array}$ \\
\hline Total dE ACCIDENTES $\ldots \ldots \ldots$ & 70 \\
\hline
\end{tabular}

En edades jóvenes (sobre todo los/as menores de un año, y en otros casos de uno a cinco años) la probabilidad, según el género, es la misma. En los accidentes de tráfico o circulación la máxima de varones es de quince a veinticuatro, y también de cuarenta y cinco a sesenta y cuatro años. En los demás accidentes, la máxima de varones es de veinticinco a cuarenta y cuatro años; y en el homicidio, lo mismo.

En cualquier caso, en los hospitales españoles mueren muy pocas personas. Apenas el 2,3 de las altas se producen por fallecimiento (eso representa 74.635 óbitos en hospitales). En la CAM la proporción llega al 3 
por 100 (es decir, 12.467 fallecidos). En números redondos, aproximadamente, sólo una de cada cinco personas que fallecen en España lo realizan dentro de un hospital. Sin embargo, la mayoría de los partos (más de 9 de cada 10) se realizan en hospital.

En resumen, se puede afirmar que la reducción de mortalidad y el progreso general en la esperanza de vida no han logrado alterar sustancialmente las diferencias del nivel de salud de la población según clases sociales, regiones o estratos rural-urbano; más bien, al contrario, el desarrollo económico - a pesar de las medidas de sanidad pública - ha creado un cierto estado de atraso sanitario relativo. Más aún, el nivel de salud de ciertos sectores de la población madrileña, en los años ochenta, se está deteriorando, a pesar (o como consecuencia) de la estructura sanitaria actual. El desarrollo económico no es suficiente para reducir la morbilidad y mortalidad en las regiones y municipios menos favorecidos, sino que es necesario montar un sistema sanitario eficaz y utilizarlo adecuadamente. Parece plausible suponer que en las etapas iniciales de desarrollo económico el crecimiento global tiene una influencia mayor sobre el nivel sanitario de la población que sobre la estructura de los servicios sanitarios. En etapas más desarrolladas, como las actuales, el nivel de salud de la población no depende tanto del crecimiento económico como de la distribución y el control de los recursos. Una política genuina de administración y distribución de recursos supone, a su vez, una «reorientación» coherente de intereses sociales; pasar de intereses de grupos (económica y políticamente) minoritarios a intereses que representan más amplios sectores. En conclusión, la influencia de las características socioeconómicas sobre el nivel de salud de la población se aminora a medida que avanza el desarrollo económico. Este proceso lleva a que el propio desarrollo económico ya no sea suficiente para solucionar los problemas de la salud y requiera un sistema sanitario que dé calidad de vida, y de aquí el planteamiento de una planificación sanitaria a nivel de la Comunidad Autónoma de Madrid.

Se trataría, por un lado, de disminuir la tasa de mortalidad, pero sin aumentar (como desgraciadamente ha sucedido a menudo) la tasa de morbilidad de la población. No se define ya como un objetivo prioritario el descenso de la mortalidad, entre otras cosas porque ha llegado ya a unas tasas difíciles de reducir, sino de mejorar el estado sanitario general de la población. Es un hecho que las tasas de mortalidad en la CAM no van a bajar mucho más, a pesar de las políticas del sector público, pero todavía pueden disminuir bastante las desigualdades y desequilibrios entre la población y la degradación medio-ambiental. Sin embargo, como objetivo manifiesto está claro que una meta sería el reducir las tasas de fecundidad y de mortalidad. Faltan modelos teóricos que permitan producir esos cambios deseables en Madrid. El cambio de la mortalidad por la morbilidad, y la sustitución de las causas infecciosas por las sociales supone un replanteamiento global del sistema sanitario durante los años del «desarrollo» que todavía no ha 
sido analizado en profundidad. Es obvia la necesidad de un estudio en detalle de las causas de muerte, y de los casos de morbilidad (tanto sean de declaración obligatoria como no), así como un análisis del medio millón de pacientes hospitalizados al año en la CAM. Es importante también realizar un estudio de la sobremortalidad masculina que se observa, sus causas y formas de evitarla. El envejecimiento gradual de la estructura de la población es otro de los temas que requieren un análisis más detallado, por sus connotaciones respecto de la salud, calidad de vida de la población y decrecimiento de la fecundidad como causa más directa de ese envejecimiento. El desarrollo de los años sesenta y setenta no ha traído un mejoramiento proporcional del nivel de salud de la población de la CAM. Los problemas sanitarios asociados, pues, a los procesos demográficos son legión. Precisamente son los que en la década de los noventa están poniendo en entredicho aquella bella frase: «de Madrid al cielo». 